\title{
Orbital evolution by dynamical tides in solar type stars
}

\section{Application to binary stars and planetary orbits}

\begin{abstract}
M. G. Witte and G. J. Savonije
Astronomical Institute "Anton Pannekoek", University of Amsterdam, Kruislaan 403, 1098 SJ Amsterdam, The Netherlands

Received 18 September 2001 / Accepted 23 January 2002

Abstract. We study the tidal evolution of eccentric binary systems consisting of a solar type main sequence star accompanied by either another solar type star, or by a planet with a mass similar to Jupiter's mass. The tidal dissipation which takes place in the solar type $\operatorname{star}(\mathrm{s})$ is calculated in the framework of dynamical tides, and resonant interaction with the $g$-mode and quasi-toroidal oscillation eigenmodes of the stellar component(s) is included in the orbital calculations. It appears that in a system of two solar type stars intervals during which harmonic components of the perturbing tidal potential become locked onto resonances with stellar oscillation modes appear ubiquitously, significantly enhancing the efficiency of the tidal coupling. In our calculations stellar binaries become circularized during the main sequence lifetime for orbital periods up to about 10 days, or 16 days in the case of very slow stellar rotation. Efficient resonance locking causes significant tidal decay in weakly eccentric planetary binaries with orbital periods up to approximately 5 days in case the solar type star is a slow rotator, and very large increase of the orbital period and eccentricity in case the star is rotating rapidly. The dynamical tide with inclusion of the effects of close resonances with the stellar oscillation modes provides considerably more efficient tidal coupling than the equilibrium tide with viscous damping of turbulent eddies.
\end{abstract}

Key words. stars: rotation - stars: oscillations - stars: binaries: close - planetary systems

\section{Introduction}

In almost all studies the tidal processes of stellar synchronisation and orbital circularization in stellar binary systems have been divided into two cases, according to the nature of the stars which undergo the tidal deformations.

On the one hand, massive main sequence (MS) stars which have convective cores and large radiative envelopes experience forced buoyancy oscillations in their envelopes. Therefore in describing the tidal process in these stars it is necessary to use a theory which accounts for dynamical effects which arise due to the tidal forcing (Zahn 1977; Savonije \& Papaloizou 1983). Extensive studies of the role played by close resonances with the eigenmodes of these early type stars during the orbital decay was published in a number of previous papers (Witte \& Savonije 1999a,b, 2001, from now on WS99a, WS99b and WS01, respectively).

Low mass MS stars and giants, on the other hand, have extended convective envelopes, and for these stars retardation of the equilibrium tide due to the viscosity of turbulent eddies in the envelope is usually assumed to be the

Send offprint requests to: M. G. Witte, e-mail: marnix@astro.uva.nl cause of the tidal torque. In the case of binaries containing giant stars, a study by Verbunt \& Phinney (1995) shows that the observed orbital parameters of 28 binaries in open clusters can indeed be understood within Zahn's formalism for viscous damping of the tidal flow. However, in the case of narrower binaries containing MS late type components tidal periods may become shorter than the turnover time of the largest eddies, presumably reducing the turbulent viscosity. Zahn \& Bouchet (1989) claim that pre-MS circularization is more efficient than circularization during the MS and that this may explain the observations. Mathieu et al. (1992) show that the cutoff period at which binary members of clusters are found in circular orbits increases with the age of the cluster, indicating that circularization occurs on the MS. Claret \& Cunha (1997) argue that, if possible pre-MS circularization is neglected, application of the revised version of the theory of turbulent viscosity which incorporates the timescale mismatch between the tidal period and the convective turnover time (Zahn 1989) yields dissipation which is two orders of magnitude too small to explain observations of late type MS binary orbits in clusters. Goodman \& Oh (1997) remark that the viscosity at higher frequencies should be more severely reduced than in Zahn's theory, significantly reducing the 
orbital decay rate before as well as during the MS. These authors argue that the gradual circularization on the MS, as it seems to follow from observations of old halo binaries, cannot be explained by turbulent convection, even if no reduction of the viscosity due to short tidal periods is accounted for at all.

It was not until recently that the possibility of excitation of $g$-modes in the radiative cores of late type MS stars was considered as a mechanism for tidal dissipation in these low mass stars. Terquem et al. (1998) calculate radiative damping of core $g$-modes as a first order perturbation on the solutions for adiabatic oscillations, and show that near resonance with a stellar eigenmode radiation diffusion is the most important dissipative effect. However, the authors estimate that close resonances with stellar eigenmodes can nevertheless be disregarded during the tidal decay of binary orbits, if the possibility of locking the companion in a stellar resonance is not considered. Goodman \& Dickson (1998) likewise show that the dynamical tide causes dissipation which is at least as strong as the dissipation caused by the equilibrium tide. These authors treat the stellar eigenmodes by taking an average over many resonances, and consider nonlinearities and effects of stellar evolution, but conclude that the dynamical tide is nonetheless too weak to explain orbital parameters of observed systems.

In previous papers (WS99b; WS01) we showed that resonance locking due to balancing of the frequency shifting tendencies of different orbital harmonics against each other, thereby causing significant resonant oscillation amplitudes for prolonged periods of time, is the most important source of tidal dissipation in eccentric early type MS binaries. In the current work we investigate whether the same holds for late type MS binaries. First we will briefly review the method of calculating orbital evolution including resonance locking as was developed previously and next we will indicate how the nonadiabatic oscillation calculations were performed to establish the stellar response to the harmonic tidal forcing. Orbital evolution calculations of late type systems using this method are presented, both for binaries consisting of two solar type stars, and for planetary binaries of a single Jupiter-like planet in close orbit about a late type MS star.

\section{Equations of tidal evolution and resonance locking}

For a more extensive description of the following we refer to WS99b and WS01.

\subsection{Evolution of orbit and stellar spin}

The orbit averaged variations of the orbital energy and angular momentum

$E_{\mathrm{orb}}=-\frac{G M_{1} M_{2}}{2 a}$ and

$H_{\mathrm{orb}}=\frac{M_{1} M_{2}}{M_{1}+M_{2}} a^{2} \omega \sqrt{1-e^{2}}$,

occurring due to the dissipation of the tidal flow induced in both of the components, are calculated assuming that the tidal perturbations of each of the stars is linear with respect to the perturbing tidal potential.

Let us define $a$ to be the semi-major axis of the relative elliptic orbit one component describes around the other, $e$ its eccentricity and $\omega=2 \pi / P_{\text {orb }}=\sqrt{\frac{G\left(M_{1}+M_{2}\right)}{a^{3}}}$ the mean orbital angular velocity of the stars, which have masses $M_{1}$ and $M_{2}$. Then, the tidal potential caused by star 2 which perturbs star 1 can be decomposed as

$$
\begin{aligned}
\Phi_{2}(r, \vartheta, \varphi, t)= & -\sum_{l=2}^{\infty} \sum_{m=0}^{l} r^{l} P_{l}^{m}(\cos \vartheta) \\
& \times \sum_{n=-\infty}^{\infty} c_{n}^{l m} \cos (n \omega t-m \varphi),
\end{aligned}
$$

with $(r, \vartheta, \varphi)$ spherical polar coordinates relative to the centre of mass of star 1 , and $P_{l}^{m}(\cos \vartheta)$ the associated Legendre functions. The coefficients

$c_{n}^{l m}=\left(\frac{G M_{2}}{a^{l+1}}\right) \epsilon_{m} \frac{(l-m) !}{(l+m) !} P_{l}^{m}\left(\cos \frac{\pi}{2}\right) h_{n}^{(l+1), m}$

are given in terms of the Hansen coefficients

$h_{n}^{(l+1), m}=\frac{1}{2 \pi} \int_{-\pi}^{\pi}\left(\frac{a}{r^{\prime}}\right)^{l+1} \mathrm{e}^{\mathrm{i}\left(m \varphi^{\prime}-k M\right)} \mathrm{d} M$,

where $M=\omega t$ is the mean anomaly and primed coordinates give the location of star 2 in its orbit; we only consider the dominant $l=2$ contribution. Each term in the sum of Eq. (3) results in an amount of dissipation characterised by the torque integral

$$
\begin{gathered}
\mathcal{T}_{1 n}^{l m}=-\pi c_{n}^{l m} \int_{0}^{R_{1}} \int_{0}^{\pi} \operatorname{Im}\left(\rho_{1}^{\prime}(r, \vartheta)\right) . \\
P_{l}^{m}(\cos \vartheta) r^{l+2} \sin \vartheta \mathrm{d} \vartheta \mathrm{d} r
\end{gathered}
$$

in which the imaginary part of the steady state density perturbation $\rho_{1}^{\prime}=\rho_{1}^{\prime}(r, \vartheta) \mathrm{e}^{\mathrm{i}(n \omega t-m \varphi)}$ of star 1 is to be calculated numerically. The secular changes of orbital energy and angular momentum due to each of these terms is given by

$\dot{E}_{1 n}^{l m}=n \omega \mathcal{T}_{1 n}^{l m} \quad$ and $\quad \dot{H}_{1 n}^{l m}=m \mathcal{T}_{1 n}^{l m}$,

with corresponding terms $\dot{E}_{2 n}^{l m}$ and $\dot{H}_{2 n}^{l m}$ for dissipation in star 2 caused by the tidal force field of star 1 . In view of the assumed linearity, the net changes of energy and angular momentum follow as

$\dot{E}_{\text {orb }}=-\sum_{i} \sum_{m} \sum_{n} \dot{E}_{i n}^{l m}=-\sum_{i} \dot{E}_{i}$

and

$\dot{H}_{\text {orb }}=-\sum_{i} \sum_{m} \sum_{n} \dot{H}_{i n}^{l m}=-\sum_{i} \dot{H}_{i}$, 
where $i=1,2$ for the two stars. From these, relations governing the rates of change of the orbital period and eccentricity can readily be established.

The rates of change of the angular spin velocities $\Omega_{i}$ are determined by the tidal secular rate of change of angular momentum $\dot{H}_{i}$, the changing moment of inertia of each of the stars $\dot{I}_{i}$ and, in some of our calculations, by the torque $\mathcal{T}_{i, \mathrm{mb}}$ on each star generated by magnetic braking. In describing the process of magnetic braking we use the prescription given by Verbunt \& Zwaan (1981):

$\mathcal{T}_{i, \mathrm{mb}}=-5 \times 10^{-30} c_{\mathrm{mb}} M_{i} R_{i}^{4} \Omega_{i}^{3}$.

For $c_{\mathrm{mb}}=1$ the canonical strength of magnetic braking is acquired, assuming for simplicity that we can take the values $k^{2}=0.1$ and $f=1$ for the parameters used in their paper.

The time evolution of the system is thus described by the relations

$$
\begin{aligned}
\frac{\mathrm{d} e^{2}}{\mathrm{~d} t} & =\frac{2 a}{G M_{1} M_{2}}\left(\left(1-e^{2}\right) \dot{E}_{\mathrm{orb}}-\omega \sqrt{1-e^{2}} \dot{H}_{\mathrm{orb}}\right) \\
\frac{1}{a} \frac{\mathrm{d} a}{\mathrm{~d} t} & =\frac{2 a}{G M_{1} M_{2}} \dot{E}_{\mathrm{orb}} \\
\frac{\mathrm{d} \Omega_{i}}{\mathrm{~d} t} & =\frac{1}{I_{i}}\left(\dot{H}_{i}+\mathcal{T}_{i, \mathrm{mb}}\right)-\frac{H_{i}}{I_{i}^{2}} \dot{I}_{i} .
\end{aligned}
$$

\subsection{Resonance locking}

Each harmonic component of the perturbing potential of the companion in its eccentric orbit excites a harmonic oscillation in star $i$. Shifting of the harmonic forcing frequency $\bar{\sigma}_{n}=n \omega-m \Omega_{i}$ relative to the frequency $\bar{\sigma}_{0, i k}$ of the eigenmode labelled $k$ of this star is given by

$$
\begin{aligned}
& \frac{\mathrm{d}}{\mathrm{d} t}\left(\bar{\sigma}_{n}-\bar{\sigma}_{0, i k}\right)=n \sum_{i^{\prime}, m^{\prime}, j} \frac{3 j}{I_{\mathrm{orb}}} \mathcal{T}_{i^{\prime} j}^{l m^{\prime}} \\
& \quad-\frac{1}{I_{i}}\left(m+\frac{\partial \bar{\sigma}_{0, i k}}{\partial \Omega_{i}}\right)\left(\mathcal{T}_{i, \mathrm{mb}}+m \sum_{m^{\prime}, j} \mathcal{T}_{i j}^{l m^{\prime}}\right) \\
& \quad+\left(\frac{\Omega_{i}}{I_{i}}\left(m+\frac{\partial \bar{\sigma}_{0, i k}}{\partial \Omega_{i}}\right) \frac{\mathrm{d} I_{i}}{\mathrm{~d} X_{i}}-\frac{\partial \bar{\sigma}_{0, i k}}{\partial X_{i}}\right) \dot{X}_{i}
\end{aligned}
$$

where $X_{i}$ is the central hydrogen abundance of star $i$. Following the procedure in WS01, we can introduce the shorthand notation

$$
\begin{aligned}
& \mathrm{d}_{t}\left(\bar{\sigma}_{n}-\bar{\sigma}_{0, i k}\right)= \\
& \quad\left[\partial_{t, n}+\partial_{t, \not x}+\partial_{t, \ddot{\gamma}}+\partial_{t, \dot{X}}+\partial_{t, \mathrm{mb}}\right]\left(\bar{\sigma}_{n}-\bar{\sigma}_{0, i k}\right)
\end{aligned}
$$

for this relation, in which the contribution of the action of magnetic braking to Eq. (13) has been contracted into

$\partial_{t, \mathrm{mb}}\left(\bar{\sigma}_{n}-\bar{\sigma}_{0, i k}\right) \equiv-\frac{1}{I_{i}}\left(m+\frac{\partial \bar{\sigma}_{0, i k}}{\partial \Omega_{i}}\right) \mathcal{T}_{i, \mathrm{mb}}$

During resonance locking the terms between the square brackets in Eq. (14) cancel for a possibly large value of the tidal torque $\mathcal{T}_{i n}^{l m}$, causing rapid orbital evolution without having the forcing frequency $\bar{\sigma}_{n}$ drift away from resonance with the stellar eigenmode at frequency $\bar{\sigma}_{0, i k}$. This way, the enhanced orbital decay may extend over a large period of time, possibly causing significant changes to the orbital parameters.

\subsection{The dynamical tide in a solar type star}

For a rotating star the stellar oscillation equations retain their separability into $r, \vartheta$ and $\varphi$ factors if the socalled traditional approximation is applied, neglecting the $\vartheta$ component of the rotational angular velocity. Thus, separate numerical solutions can be calculated for the $\vartheta$ and $r$ functions, eliminating the need for numerically very intensive 2D calculations (see Savonije \& Papaloizou 1997). For fourteen stellar models along the MS it has therefore been possible to calculate the very dense torque spectra of $g$ - and (quasi-toroidal) $q$-modes occurring in the radiative cores of these stars up to very high $(>1000)$ numbers of radial nodes, for modest uniform rotation speeds of the stars (see Savonije \& Witte 2002, from now on SW02). Additionally, the excitation at low forcing frequencies of inertial modes in the convective envelope of such a star provides a smooth torque level with a strong broad peak. Interpolation within the results of the calculations presented in SW02 provides the torques needed to conduct the orbital calculations for the current study.

Only forcing due to the dominant tidal harmonic with spherical degree $l=2$ and $m=2$ or $m=0$ is considered. The response of the rotating star is acquired by first solving an eigenvalue problem in the $\vartheta$ coordinate, after which the acquired eigenvalue $\lambda_{n}$ is substituted into the radial equations, which are solved next. Only for the lowest order latitudinal eigenvalue $\lambda_{1}$ torques are calculated. The torque spectra which are used for the orbital calculations are thus overall the strongest, however because of the strongly enhanced torque near a stellar eigenfrequency, oscillations with eigenvalues $\lambda_{n}$ of higher orders $n>1$, possibly excited by the higher degree orbital harmonics with $l>2$, could, near an eigenfrequency of such a mode, give rise to a larger torque than the dominant oscillation mode at the same frequency. In the $2 \mathrm{D}$ calculations all the orders $n$ for the angular eigenvalue $\lambda_{n}$ were present (though calculated with very limited $\vartheta$ resolution), but in the current work we neglect the higher orders.

\section{Numerical results}

Due to the very dense spectra of $g$ - and $q$-modes in solar type stars, the tidal evolution of the orbit of a binary containing one or two of these stars involves very many resonance crossings, and the chances of encountering resonance locking should be quite large.

In this respect we should consider the order $n$ of the harmonic component which has an almost neutral frequency shifting property, i.e. for which the self-shift $\partial_{t, n}\left(\bar{\sigma}_{n}-\bar{\sigma}_{0, i k}\right) \equiv \zeta_{i n n} \mathcal{T}_{i n}^{l m}$ vanishes; $\zeta_{i n n} \simeq 0$. Harmonics with orders close to this $n$ have a very weak tendency 
of driving themselves out of resonance, and for such harmonics one expects the most effective resonance locking, at large amplitudes of oscillation. Noting that for this order we have $n \simeq m \sqrt{I_{\text {orb }} /\left(3 I_{i}\right)}$, and substituting $I_{i} \simeq 7 \times 10^{53} \mathrm{~g} \mathrm{~cm}^{2}$ and, for a system of two solar type stars, $I_{\text {orb }} \simeq 3 \times 10^{56} P_{\text {orb }}^{\frac{4}{3}} \mathrm{~g} \mathrm{~cm}^{2}$ (with $P_{\text {orb }}$ in days), we typically find $n \simeq 10^{2}$. Moderately eccentric orbits do not contain significant harmonics of such high orders, therefore we do not expect resonance locking to take place near this point of optimum efficiency. On the other hand, a larger negative value of $\zeta_{i n n}$ for the stronger harmonics of order $n \simeq 10^{1}$ implies that the torque which is generated by the resonant excitation during locking need not be as large in order to neutralise the terms in Eq. (14), i.e. locking can take place on weaker eigenmodes of the star, or already further down from the peak torque of a strong mode. In combination with the large number of eigenmodes in the stellar spectra, this means that locked conditions in dual MS star binaries should very easily be attained, and should be able to last for long timespans.

For a planetary binary with a $1 M_{\odot}$ star and a Jupiter-like $\frac{1}{1000} M_{\odot}$ planet, on the other hand, we find $I_{\text {orb }} \simeq 2 \times 10^{53} P_{\text {orb }}^{\frac{4}{3}} \mathrm{~g} \mathrm{~cm}^{2}$, so in this case neutral frequency shifting occurs at frequencies near $n \simeq 10^{0}$. Strong tidal harmonics may therefore show very efficient locking, while nearby strong forcing frequencies could have different signs for $\zeta_{i n n}$, potentially leading to quite complex orbital evolution.

We will first examine binaries consisting of two MS stars, and afterwards turn attention to planetary binaries.

\subsection{Stellar binaries}

\subsubsection{A moderately wide, moderately eccentric orbit}

As an example of tidal evolution in binaries containing two solar type stars, we describe the evolution of a binary with an orbit of initially $P_{\text {orb }}=16$ days and with initial eccentricity $e=0.6$. From now on, at the beginning of the calculations star 1 will be taken to have a core hydrogen abundance of $X_{\mathrm{c}}=0.689$, while its companion, if also of stellar type, starts at $X_{\mathrm{c}}=0.68$.

To prevent that extrapolation of the linear oscillation calculations into the nonlinear regime for large amplitudes yields unrealistically large tidal torque values, the tidal torques are, when necessary, scaled down such that the oscillation speed near the stellar centre does not exceed the local speed of sound. This refinement turns out to be of little influence on the orbital decay calculations, because the tidally forced oscillations which cause the most significant dissipation have only moderate amplitudes.

At $t=0$ the stars are taken to rotate at $20 \%$ of their respective breakup frequencies (corresponding to rotation periods of approximately 12 hours). Figure 1 shows the resulting evolution taking place during the first $10^{8}$ years. Since the time evolution of the rotation speeds of both stars is very similar, only the rotation velocity of star 1 in units of the orbital angular velocity at periastron $\left(\omega_{\text {per }}=\right.$ $\sqrt{\frac{1+e}{(1-e)^{3}}} \omega$ ) is shown (panel 1d). Figure 2 indicates how the frequencies $\sigma=\bar{\sigma}+m \Omega_{\mathrm{s}}$ of eigenmodes of star 1 in a nonrotating frame (solid curves) drift relative to the orbital harmonic frequencies $\sigma_{n}=n \omega$ (horizontal dashed lines). Curves with the resonance frequencies of various pro- and retrograde $g$-modes with radial orders $k$ are labelled $g_{ \pm k}$, the retrograde $q$-modes are localised between the two curves labelled as $q_{k}$. The fat dashed line close to the upper $q$-mode curve gives the frequency for which $\bar{\sigma}=0$; horizontal fat line elements show the forcing frequencies at which resonance locking takes place.

Although no magnetic braking is included in this calculation $\left(c_{\mathrm{mb}}=0\right.$, see Eq. $\left.(9)\right)$, strong retrograde forcing rapidly spins the stars down during the first few tens of millions of years ( $t \lesssim 0.08 \mathrm{Gyr}$ ), causing many resonance crossings (see Fig. 2) which result in rapid variations of the timescale of eccentricity changes (panel 1c). During this period the orbit expands by a small amount (see panels $1 \mathrm{a}$ and $1 \mathrm{~b} ; \Delta e \approx 0.005, \Delta P_{\text {orb }} \approx 0.6 \mathrm{~d}$ ). Due to repetitive crossing of the broad resonance peak of the retrograde inertial modes, the star is seen to spin down at a fluctuating rate, and orbital period and eccentricity increase erratically. From $t \simeq 0.08 \mathrm{Gyr}$ onwards prograde resonance locking takes place (see Fig. 2), reducing the spin-down rate of the stars (see panel 1d), and thereby the rate at which resonances are crossed (see panel 1c). From this moment on the orbit decays due to the dissipation taking place at high forcing frequencies $(n \simeq 18)$ for which resonance locking occurs. Though the thick line segments indicating resonance locking in Fig. 2 appear to stretch uninterruptedly over large intervals of time, they in fact consist of many short sections in quick succession, where each adjacent piece corresponds to resonance locking on the next $g$-mode one down in radial order.

Figure 3 gives the continuation of the evolution in Fig. 1, showing the evolution until circularization and corotation have been established near the end of the MS lifetime of the stars $(t \simeq 11 \mathrm{Gyr})$. Figure 4 shows the corresponding resonance shifting and locking during the first few billion years of this continued evolution.

The rapid stellar spin down which starts immediately at $t=0$ continues at a decreased rate (see Fig. $1 \mathrm{~d}$ ) during the prograde resonance locking phase (mainly due to orbital harmonic $n=18$ ) which commences around $t=0.08 \mathrm{Gyr}$, until around $t=0.5 \mathrm{Gyr}$ the star is spun down to nearly quasi-synchronism $\left(\Omega_{1} \approx \omega_{\text {per }}\right)$, see Fig. 3d. In Fig. 4 the stellar spin down occurring up to $t=0.5$ Gyr causes the dashed line corresponding to $\bar{\sigma}=0$ to come down, so that more and more retrograde harmonics (which spin the star down) become prograde. At $t=0.5$ Gyr the harmonic with $n=7$ has moved through the $q$-mode range and is close to becoming prograde, and its retrograde forcing torque diminishes as it approaches $\bar{\sigma}=0$. As a result the total retrograde forcing due to the orbital harmonics with $n \leq 7$ diminishes and the stellar spin down halts, preventing the $n=7$ harmonic from 

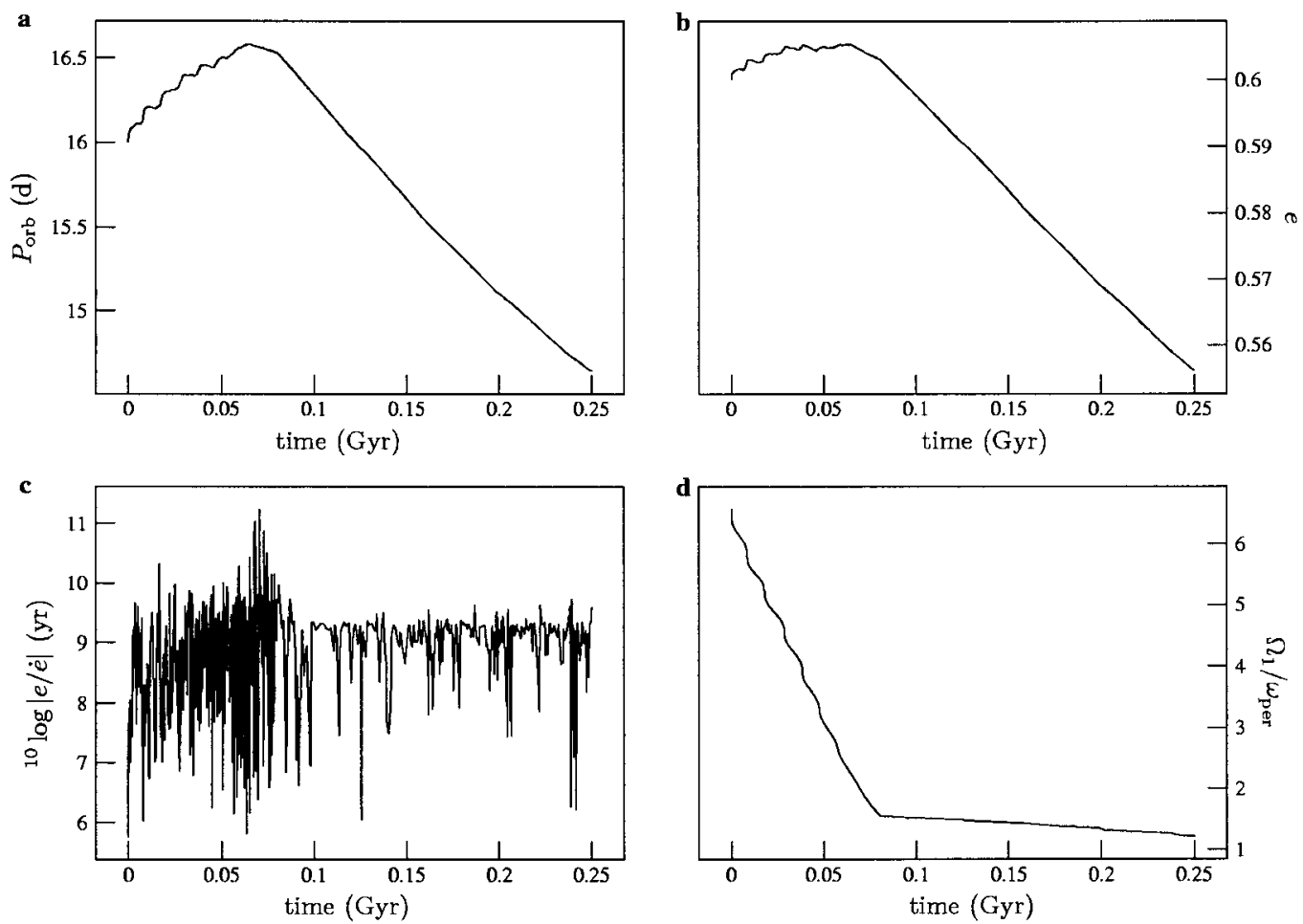

Fig. 1. Orbital evolution of a system of two $1 M_{\odot}$ MS stars; a) orbital period, b) orbital eccentricity, c) timescale of eccentricity changes, d) rotation of star 1 in units of orbital angular velocity at periastron. After $t \simeq 0.035$ Gyr resonance locking takes place.

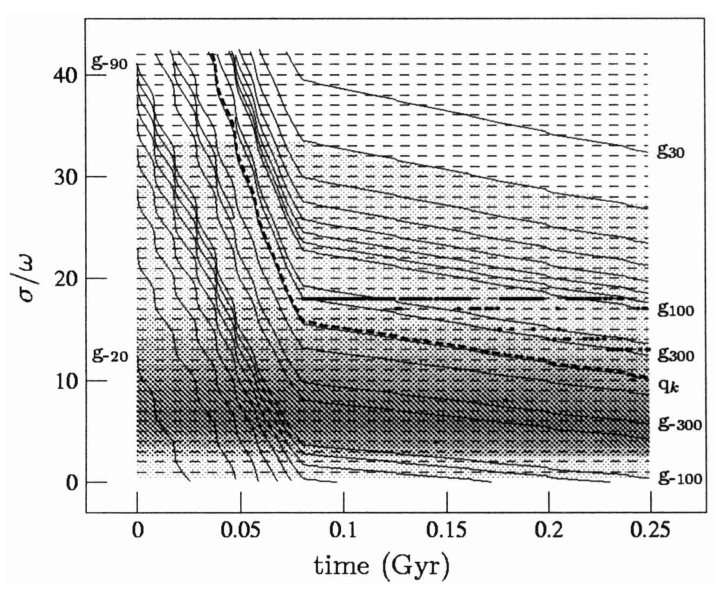

Fig. 2. The resonance frequencies of star 1 in the inertial frame (solid curves) as a function of time shown here relative to the frequencies of the orbital harmonics (horizontal dashed lines). Thick line segments indicate resonance locking. The thick dashed curve divides pro- and retrograde forcing frequencies. Greyscale shading indicates the strength of each orbital harmonic $n$. The evolution corresponds to that shown in Fig. 1.

becoming prograde. For the next 0.4 Gyr (until $t \simeq$ $0.9 \mathrm{Gyr}$ ) the star spins up due to the now dominant prograde harmonics, eventually reaching a rotation speed of $\simeq 1.4 \omega_{\text {per }}$ again (panel $3 \mathrm{~d}$ ). The rate at which the (inertial) frequency corresponding to $\bar{\sigma}=0$ increases due to this stellar spin up is balanced by the rate at which the frequency of harmonic $n=7$ increases due to the orbital decay (increase of $\omega$ ), such that harmonic $n=7$ remains close to $\bar{\sigma}=0$. At $t \simeq 0.9$ Gyr the increasing orbital frequency causes harmonic $n=6$ to reach the lowest radial order $q$-mode, whose spin down effect on the star is greatly enhanced as a result. The stellar spin up is thus reversed into down spin again, forcing $n=7$ now to become prograde. This sequence of delayed crossing of one harmonic from retrograde to prograde frequency, eventually enforced by enhanced retrograde forcing due to $q$-modes or resonance locked retrograde $g$-modes repeats a number of times, causing the stellar spin to fluctuate around a level (panel 3d) which becomes lower as the peak of the Hansen distribution of orbital harmonics shifts to lower frequencies (shading in Fig. 4) as the orbital eccentricity decreases (panel 3b).

Resonance locking is very common in this system. At any time during the interval shown in Fig. 4, at least one orbital harmonic is locked to a prograde $g$-mode resonance of the star. Although the torque peak associated with the inertial modes is much wider than $g$-mode resonance peaks of comparable frequencies, it reaches a smaller peak torque value, therefore resonance locking onto inertial modes does not occur. If we consider the resonance locking balance Eq. (14) for a locked harmonic $n$, retaining only the dynamical terms proportional to tidal torque integrals $\mathcal{T}$ (neglecting effects of stellar evolution), we can write

$$
\zeta_{n n} \mathcal{T}_{n}+\sum_{j \neq n} \zeta_{n j} \mathcal{T}_{j}=0
$$



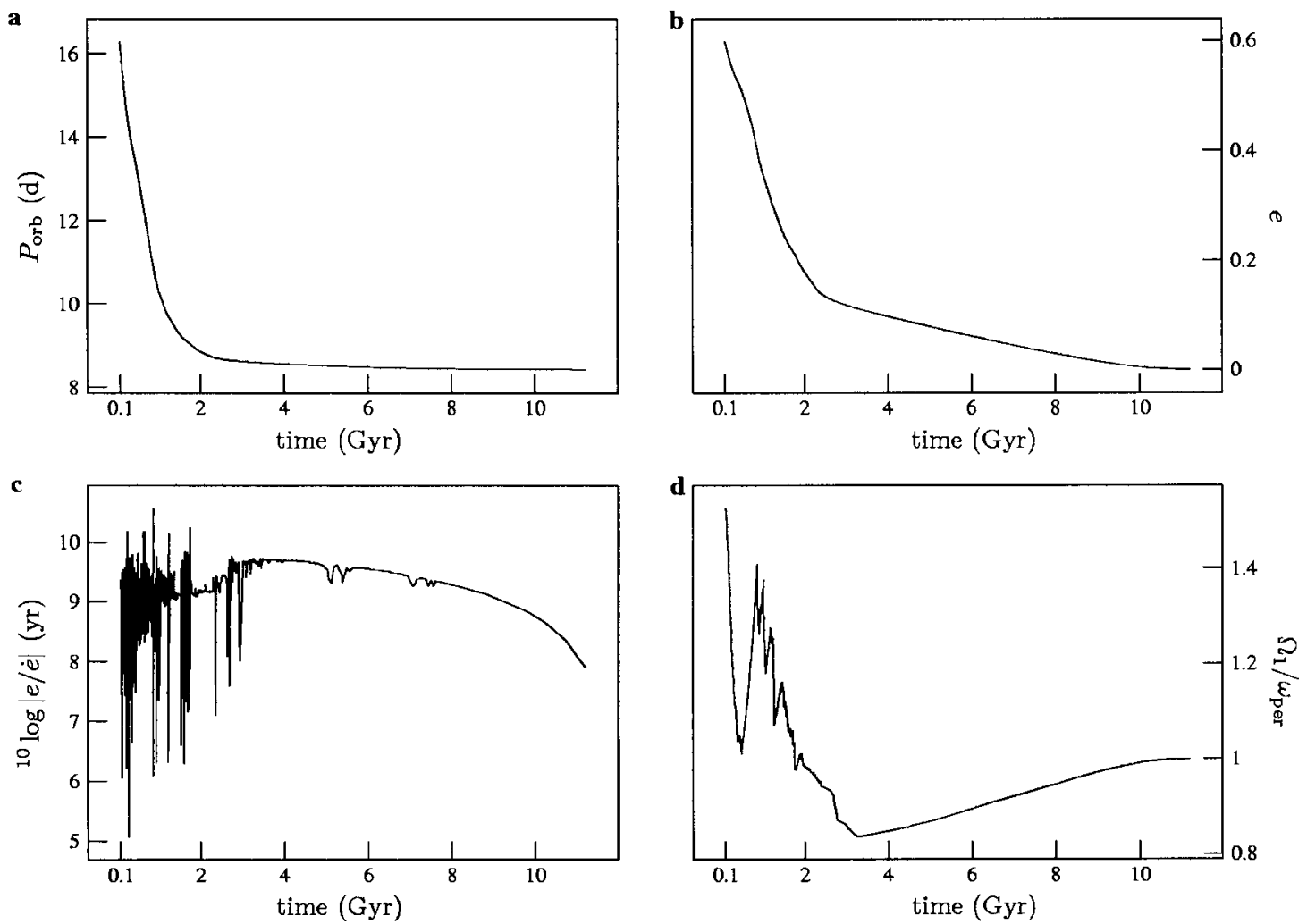

Fig. 3. Orbital evolution after $t=0.1 \mathrm{Gyr}$. At the end of the MS, circularization and corotation are established.

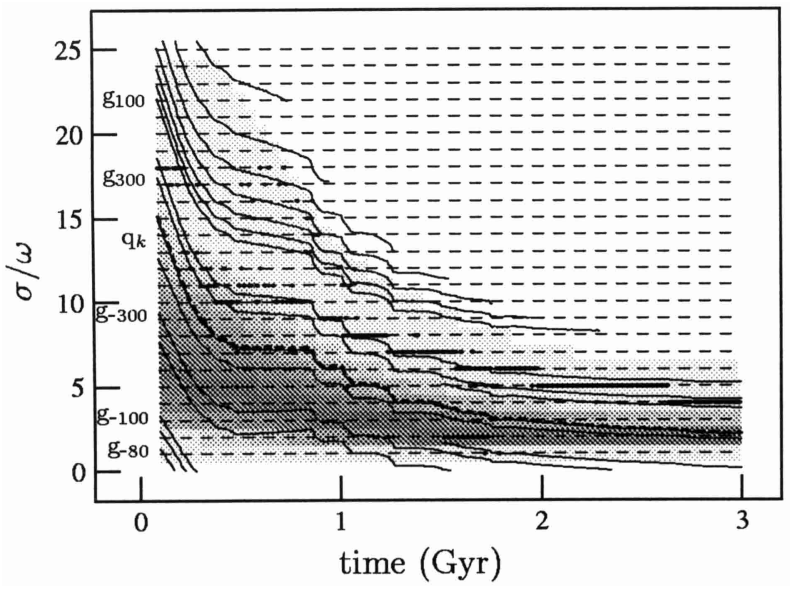

Fig. 4. Same as Fig. 2 but now corresponding to Fig. 3. During the first part of the evolution resonance locking is established during the entire period. The solid curves are truncated when the corresponding orbital harmonic has become too weak.

The resonant torque integral needed to establish locking

$\mathcal{T}_{n}=-\sum_{j \neq n} \frac{\zeta_{n j}}{\zeta_{n n}} \mathcal{T}_{j}$

is rather strong and prograde, since most of the other strong torque integrals $\mathcal{T}_{j}$ which occur for small values of $j$ near the strongest Hansen coefficients are retrograde. The fraction $\frac{\zeta_{n j}}{\zeta_{n n}}$ is large if resonance locking would occur near the value of $n$ for which $\zeta_{n n}=0$, but is closer to unity in the current case, for which $n$ is smaller. The tidal energy exchange rate resulting from this locking follows as

$$
\begin{aligned}
\dot{E}_{\text {orb }} & =-\sum_{j} j \omega \mathcal{T}_{j}=-n \omega \mathcal{T}_{n}-\sum_{j \neq n} j \omega \mathcal{T}_{j} \\
& =\sum_{j \neq n}\left(n \frac{\zeta_{n j}}{\zeta_{n n}}-j\right) \omega \mathcal{T}_{j} .
\end{aligned}
$$

The high order $n$ of the locked harmonic compared to the low orders $j$ of the mainly retrograde torque integrals which drive the locking thus ensures efficient orbital decay.

When the eccentricity decreases and the retrograde forcing diminishes resonance locking becomes less intense and the orbital decay slows down ( $t \gtrsim 3$ Gyr). However towards the end of the main sequence ( $t \lesssim 11$ Gyr), expansion of the stars shrinks their relative separation $\frac{a}{R_{i}}$, causing the tidal timescale to shorten again (panel 3c).

This example indicates that the tidal evolution of solar type stellar binaries is governed by the resonant interaction with the stellar eigenmodes of oscillation, through the mechanism of resonance locking. For rapidly rotating stars the exact initial rotation speed is not significant, since rapidly rotating stars will quickly spin down until resonance locking commences; during the initial stellar spin down only small orbital changes occur.

A large number of resonance crossings and intervals of resonance locking occur during the evolution, and it should be obvious that the exact sequence of crossings and lockings which occurs is very sensitive to the exact starting parameters of the system. A small deviation near 


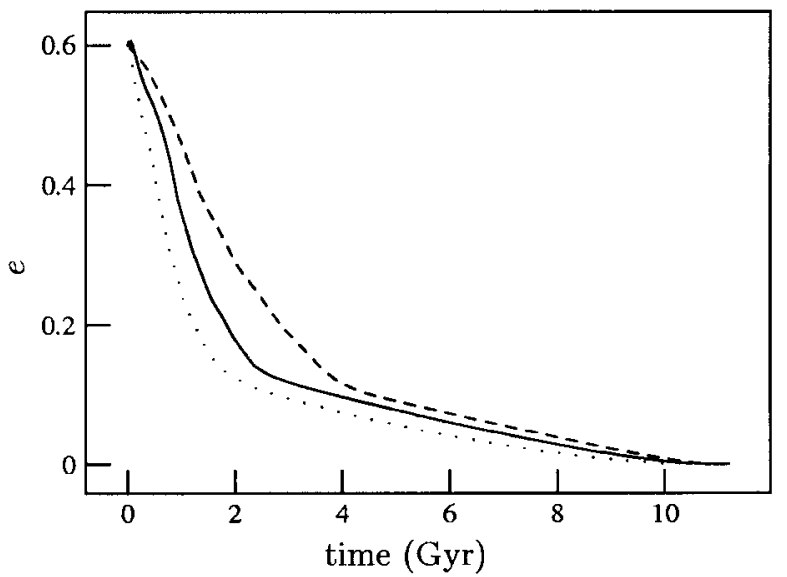

Fig. 5. Inclusion of magnetic braking intensifies the resonance locking, leading to more rapid orbital decay (dotted line) than the case in which magnetic braking is not taken into account (solid line). If the torque due to inertial modes is eliminated, the reduced spin down of the star leads to less efficient resonance locking and slower orbital decay (dashed line).

the beginning of the evolution might result in a different sequence of events later on. However, due to the dense distribution of resonance peaks in the torque spectrum of a solar type star, and due to the small differences in width and height between two adjacent peaks (see SW02), similar orbital evolution results if starting conditions are similar. The influence of each individual resonance is only small, and after very many crossings and instances of resonance locking the evolution depends only on the global distribution of the resonance peaks, not on the exact sequence of events. Later on (see Sect. 3.1.4) this will enable us to study the circularization cutoff period of binaries in clusters by calculating multiple evolutionary tracks with starting conditions close together.

The accuracy with which the evolutionary equations are solved, i.e. the size of the timesteps which is chosen, can also be of influence on the way the evolution takes place. E.g., the duration of resonance locking may be sensitive to the accuracy of the calculation. Indeed, a recalculation of the evolution pictured in Fig. 3 with considerably less stringent conditions on the required accuracy during each timestep results in somewhat different evolution. In graphs of this calculation (not printed) the difference can be seen from the timescale in panel $3 \mathrm{c}$, in which the distribution of plotted peaks is a random combination of resonance peaks and print resolution, and from the stellar rotation in panel $3 \mathrm{~d}$, in which the distinct jags have somewhat different sizes and shapes due to different timing of e.g. strong $q$-mode crossings. Globally, however, the evolution is very much alike, and in fact the evolution of the orbital period and eccentricity (panels $3 \mathrm{a}$ and $3 \mathrm{~b}$ ) can not be distinguished from one calculation to the other. We choose the accuracy during each timestep such that even the evolution of the stellar spin only changes insignificantly if the accuracy is varied by a small amount.

\subsubsection{Magnetic braking and inertial modes}

To determine how magnetic braking can modify the tidal evolution of a system with stars that have high rotation rates, we repeat the evolution of the system with $P_{\text {orb }}=16$ days, $e=0.6$ and $\Omega_{i}=0.2 \omega_{\mathrm{c}}$, thereby setting the coefficient of magnetic braking to unity $\left(c_{\mathrm{mb}}=1\right)$. Figure 5 shows the resulting evolution of the orbital eccentricity with a dotted line, together with a solid line for the original evolution without magnetic braking. Additionally, the evolution which results if the torque due to inertial modes in the envelope is disregarded (and no magnetic braking is taken into account) is plotted with a dashed line. As is to be expected, the increased spin down of the stars due to magnetic braking causes resonance locking to become more intense, leading to faster orbital decay during the first few Gyrs. Disregarding the inertial mode torque, on the other hand, eliminates much of the response caused by the strong orbital harmonics which excite retrograde modes in the stars; for low retrograde forcing frequencies the tidal coupling with retrograde $g$-modes is weak, see Fig. 2 of SW02. The resulting orbital decay (dashed line) is therefore slower; the weaker retrograde forcing results in less intense resonance locking. Magnetic braking and inertial mode torques thus have similar effects of comparable strength on this type of system. For the mechanism of magnetic braking no fundamental theory is available, although its occurrence can be qualitatively understood. Its parametric fit to observational data could be subject to future refinements in the interpretation of the data, or might change as more data becomes available. However, even if the effectiveness of magnetic braking proves to be much less than what is currently believed, the excitation of inertial modes in the convective envelopes of solar type binary stars seems an effective alternative for rapid down spin. We will not include magnetic braking unless specifically stated otherwise.

Towards the end of the evolution the stellar spin frequencies have decreased significantly $\left(\Omega_{i} \simeq 10^{-2} \Omega_{\mathrm{c}}\right)$, practically eliminating the differences between the different cases; without rotation neither magnetic braking nor inertial modes are active. The transition from enhanced tidal interaction due to resonance locking into slower nonresonant decay takes place for comparable eccentricities in the three cases $(e \simeq 0.1)$, but at different evolution times ranging from 2 to $4 \mathrm{Gyr}$. The three tracks of orbital evolution converge towards the end of the main sequence.

\subsubsection{Slowly rotating components}

Apparently, as long as the initial rotation of the stars is highly supersynchronous at periastron, their exact rotation is not significant. What, however, if the stars are slow rotators, starting the evolution at subsynchronous rotation? We repeat the orbital evolution of Sect. 3.1.1, but with stars which initially have rotation periods of 30 days.

Figure 6 shows how the orbital decay takes place in this case. First a short phase $(\$ 1 \mathrm{Myr})$ of fast stellar spin up 
$\mathbf{a}$
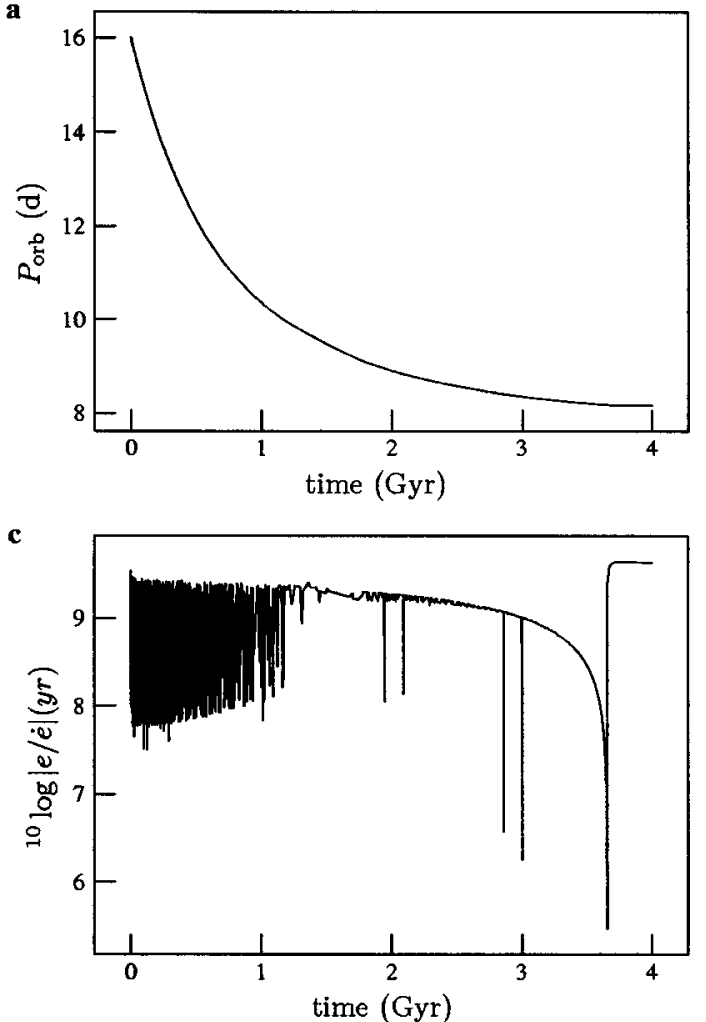
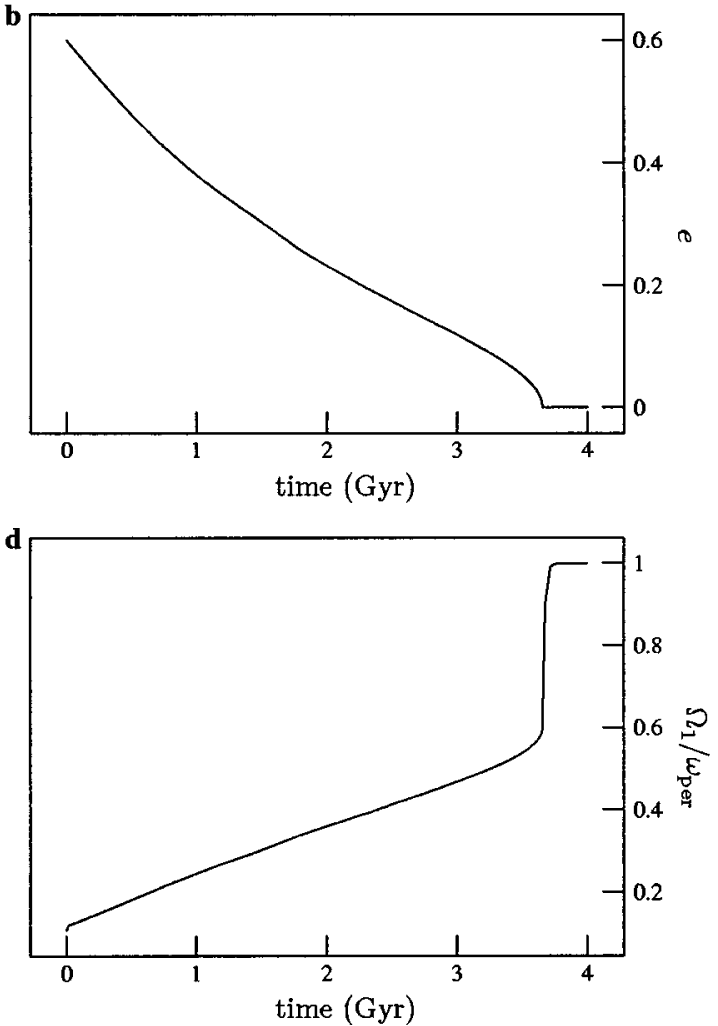

Fig. 6. Orbital evolution of a system consisting of two $1 M_{\odot}$ stars which initially have rotation periods $P_{i}=30$ days. Extensive $q$-mode locking (see Fig. 7) keeps the stellar rotation down, and circularizes the orbit within 4 Gyr.

due to forcing by the strong orbital harmonics which are all prograde for this slow stellar rotation causes the retrograde but intrinsically weak $n=1$ harmonic to be driven down towards the strong $q$-mode resonances. Once this harmonic reaches the $q$-mode with 55 radial nodes it becomes locked (Fig. 7), decreasing the stellar spin up rate. The $n=1$ retrograde $q$-mode locking is extremely stable and lasts throughout the remaining orbital evolution of the system. From $t \simeq 0.7$ Gyr to 2.6 Gyr prograde locking on $3 \leq n \leq 6$ accelerates the orbital decay somewhat, but for the evolution of this system locking on the very strong $q$-modes remains dominant. In this calculation, circularization is reached before corotation. Once the magnitude of the Hansen coefficient with $n=1$ becomes negligible due to the declining eccentricity, the retrograde locking which keeps the stellar rotation down finally ceases. After this corotation is quickly established as well.

Comparing with the results for fast spinning stars (Fig. 3), the orbital decay rate of the slowly rotating stars is seen to be comparable until the eccentricity decayed to $e \simeq 0.1$. But whereas the decay of the fast rotators slows down after this due to the decreasing efficiency of the resonance locking, in the case of slowly rotating stars the continuing retrograde $q$-mode locking quickly forces the orbit into circularity. During this short phase only the locked retrograde harmonic $n=1$ and the dominant prograde harmonic $n=2$ are important; for the weak harmonics $n \geq 3$ the non-resonant response does not generate a significant torque. The locking balance then requires $\zeta_{11} \mathcal{T}_{1}+\zeta_{12} \mathcal{T}_{2}=0 \Rightarrow \mathcal{T}_{1}=-\zeta_{12} / \zeta_{11} \mathcal{T}_{2}$. The rate of eccentricity decay follows as

$$
\begin{aligned}
\frac{\mathrm{d} e^{2}}{\mathrm{~d} t} & =\sum_{n} \frac{\omega \sqrt{1-e^{2}}}{E_{\mathrm{orb}}}\left(n \sqrt{1-e^{2}}-m\right) \mathcal{T}_{n} \\
& =\sum_{n} \frac{\omega}{E_{\mathrm{orb}}}(n-m) \mathcal{T}_{n}+O\left(e^{2}\right) \\
& =-\frac{\omega}{E_{\mathrm{orb}}} \mathcal{T}_{1}+O\left(e^{2}\right) \\
& =\frac{\omega}{E_{\mathrm{orb}}} \frac{\zeta_{12}}{\zeta_{11}} \mathcal{T}_{2}+O\left(e^{2}\right)
\end{aligned}
$$

The torque integral $\mathcal{T}_{2}$ contains Hansen coefficient $h_{2}$ which is constant to first order in $e$ near $e=0$. The non-resonant torque integral $\mathcal{T}_{2}$ therefore varies only little during the final stages of circularization, and we find

$\frac{\mathrm{d} e^{2}}{\mathrm{~d} t} \simeq$ const. $\Rightarrow e \propto \sqrt{t_{\text {circ }}-t}$,

approximately. Here, $t_{\text {circ }}$ is the moment circularization would become established if the locking would endure up to the end. This illustrates why in the case of slowly rotating stars the rate of eccentricity decay increases towards the end of the orbital decay, as long as the $q$-mode locking remains intact.

Calculations of tidal evolution which is governed by the interaction with $q$-mode resonances may suffer from imperfections in the determination of the $q$-mode response. Our linearised calculations yield very weakly damped 


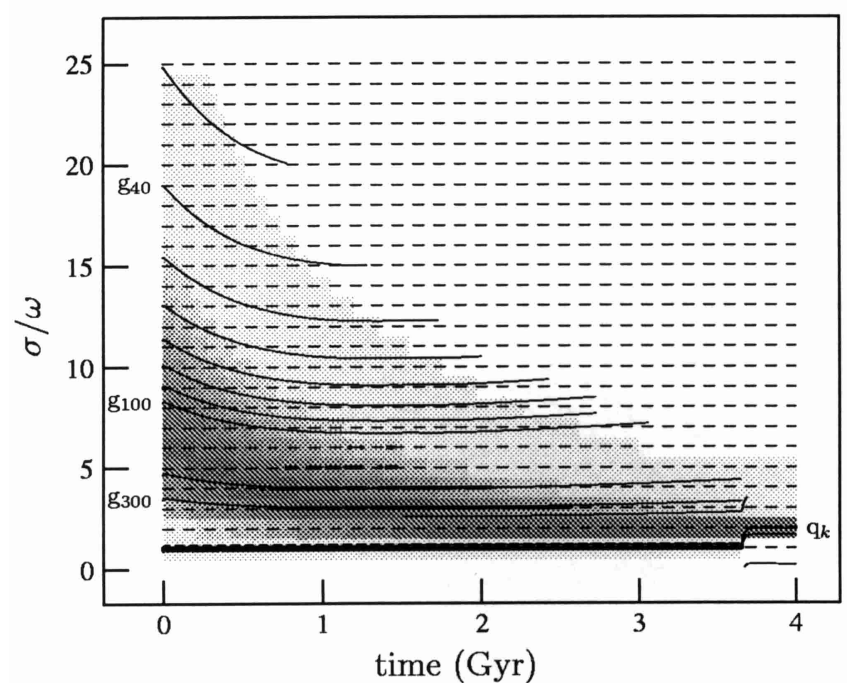

Fig. 7. In the system with slowly rotating stars, resonance locking of $n=1$ on retrograde $q$-modes is dominant.

modes, with high and narrow resonance torque peaks. Even weak additional damping, e.g. due to nonlinear processes or differential rotation could result in significantly lower and wider resonance peaks. Recalculation of the orbital evolution with the $q$-mode resonance peaks lowered and widened by an order of magnitude does not, however, give significantly different results from what is shown in Fig. 6. During resonance locking the difference between the case of narrow peaks and the case of wider peaks is only the very small difference in frequency at which the locking occurs, and this difference is much too small to cause differing orbital evolution. The moment at which $q$-mode locking is finally terminated does depend on the maximum peak height of the $q$-modes, however because the locking does not end until the locked Hansen coefficient $h_{1}$ has become very small $\left(h_{1}^{2} \simeq 10^{-6}\right.$ for $e \simeq 2 \times 10^{-3}$ ), lowering of the $q$-modes by one order of magnitude only results in the locking being terminated for $h_{1}^{2} \simeq 10^{-5}\left(e \simeq 6 \times 10^{-3}\right)$, which is still very small. Consequently, no different tidal evolution results from introducing (limited) variations of the $q$-mode damping.

\subsubsection{The circularization cutoff period}

A useful criterion for the strength of the tidal interaction is the circularization cutoff period, i.e. the largest orbital period at which circularization can be established during the main sequence. Observations of cluster binaries yield observational cutoff periods, against which the theoretical calculations can be checked.

To determine the cutoff period for the dynamical theory presented in this paper, we follow the tidal orbital decay for a large number of binary systems. Figure 8 shows the orbital evolution of all these systems evolving off the MS. In panel 8a the starting locations of the systems lie on a rectangular grid in $e-\log P_{\text {orb }}$ space, with dashed lines tracking the evolution of the systems towards the situation at $t=0.1 \mathrm{Gyr}$, as given by the solid dots. The very regular shape of the evolutionary tracks reflects the large ratio of orbital to stellar moment of inertia, implying that the orbital evolution takes place essentially at constant orbital angular momentum. The rotation of the stars at $t=0$ is taken to be $2 \omega_{\text {per }}$, such that a short period of stellar spin down is expected quickly to be followed by resonance locking, after which the evolution can be expected to proceed typically for the given orbital parameters. Some tracks which should have started at the upper left hand corner of the diagram are missing; for these cases the stellar rotation would have become larger than $20 \%$ of breakup, which is the largest value for which stellar resonance spectra have been determined. Panels 8b, $c$ and $d$ give the changes that have taken place since the previous panel for $t=0.8 \mathrm{Gyr}, t=4 \mathrm{Gyr}$ and $t=11 \mathrm{Gyr}$, respectively. Fully circularized systems with periods up to 10 days are found after 11 Gyr, while systems with initial periods up to 40 days $\left(e=0.8\right.$ in case $P_{\text {orb }}=40 \mathrm{~d}$, solid curve) are seen to circularize on the main sequence.

Inclusion of magnetic braking in these systems would probably speed up the early stages of the evolution, however the discussion in Sect. 3.1.2 indicates that no large difference in the final cutoff period is to be expected.

Starting with slowly rotating stars instead of with fast rotators, however, the $q$-mode locking on harmonic $n=1$ becomes important, typically leading to quicker orbital decay, and longer cutoff periods. Figure 9 shows the evolution of a large number of systems which at $t=0$ contain two stars with orbital periods $P_{i}=100$ days. In these calculations a short $(\sim \mathrm{Myr})$ initial interval of stellar spin up, during which very little orbital evolution occurs, brings the system into $q$-mode locking. Provided that care is taken that the $n=1$ harmonic is able to reach the $q$-modes, the rate of initial rotation is irrelevant (for $P_{i} \simeq 30$ days the results are basically the same). The buildup of systems with small but non-vanishing eccentricities, as is seen in systems with fast rotators (Fig. 8), does not occur in this case, because orbits which reach eccentricity $e \simeq 0.1$ are quickly circularized. Compared to Fig. 8 the cutoff period is larger for each time frame, at the end of the MS the cutoff period reaches approximately 16 days, orbits with $e=0.8$ up to 50 days (solid curve) circularize on the MS.

\subsubsection{Time evolution of the cutoff period}

By combining observations of clusters with populations of various ages, Mathieu et al. (1992) were able to acquire an indication of the way the circularization cutoff period changes with the age of a cluster. From left to right, the lower limits plotted in Fig. 10 give the cutoff periods for a pre-MS population of stars, for the Pleiades, the Hyades combined with Praesepe, M 67 and Halo binaries. For reference, the dotted line (without dashes) gives an estimate of the cutoff period based on calculations performed by Claret \& Cunha (1997), using the revised equilibrium tide 

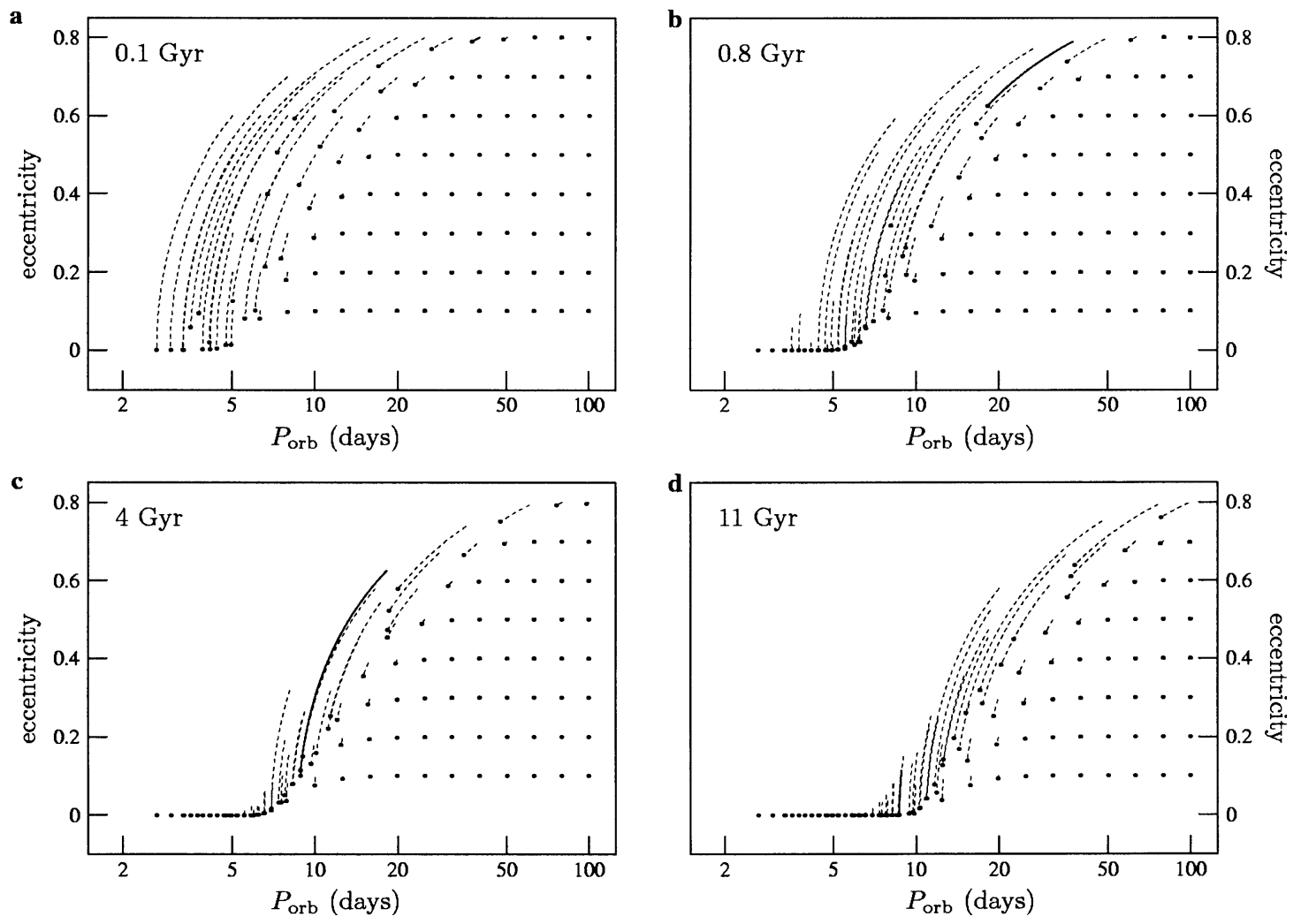

Fig. 8. Time slices of the orbital evolution of a large number of systems starting with stars rotating at twice the orbital angular velocity at periastron: $\Omega_{i}=2 \omega_{\text {per }}$. The cutoff period at the end of the MS is approximately 10 days.
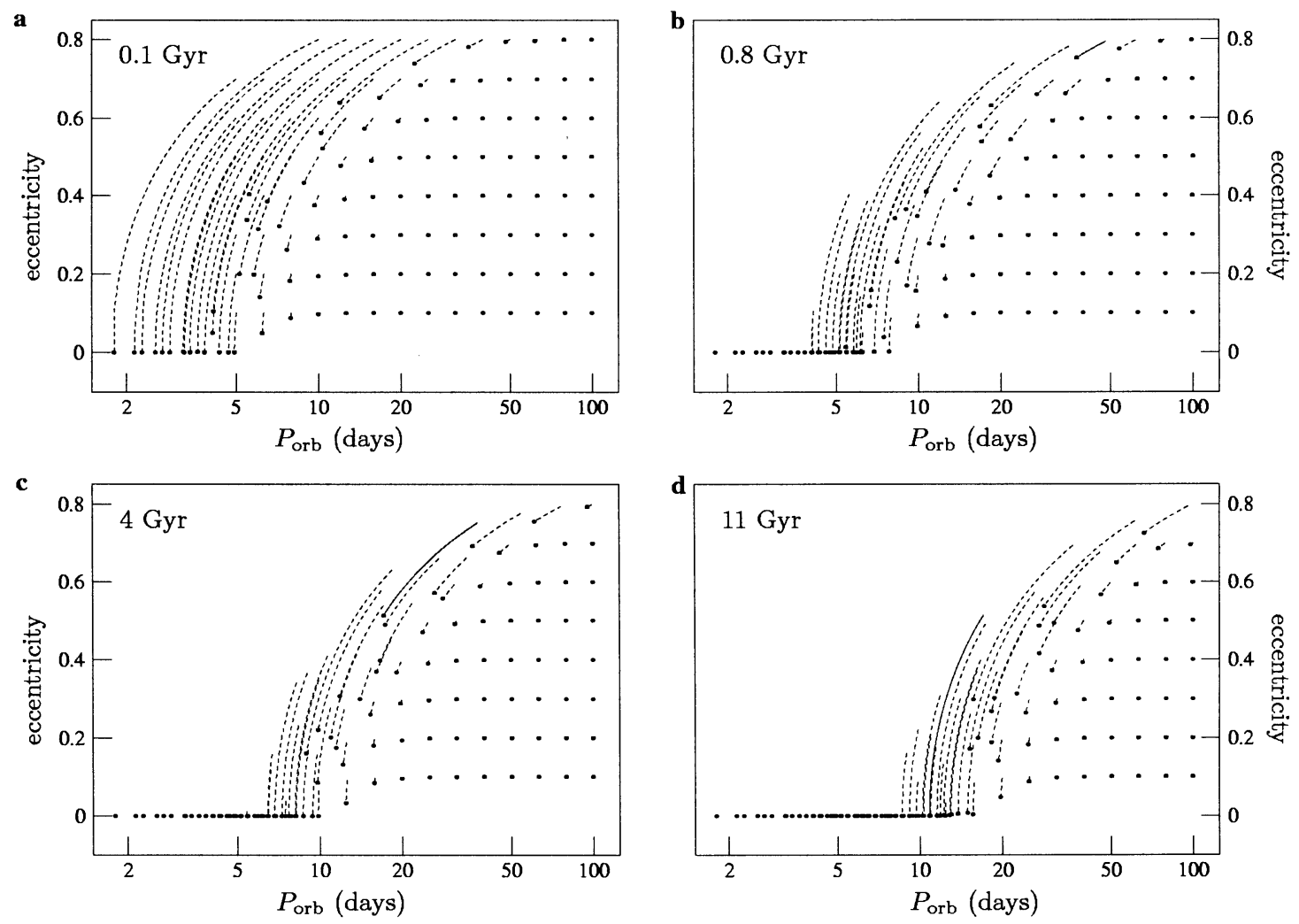

Fig. 9. For stars which rotate very slowly at $t=0 q$-mode resonance locking results in a longer cutoff period of $P_{\text {cutoff }} \simeq 16$ days after 11 Gyr. 


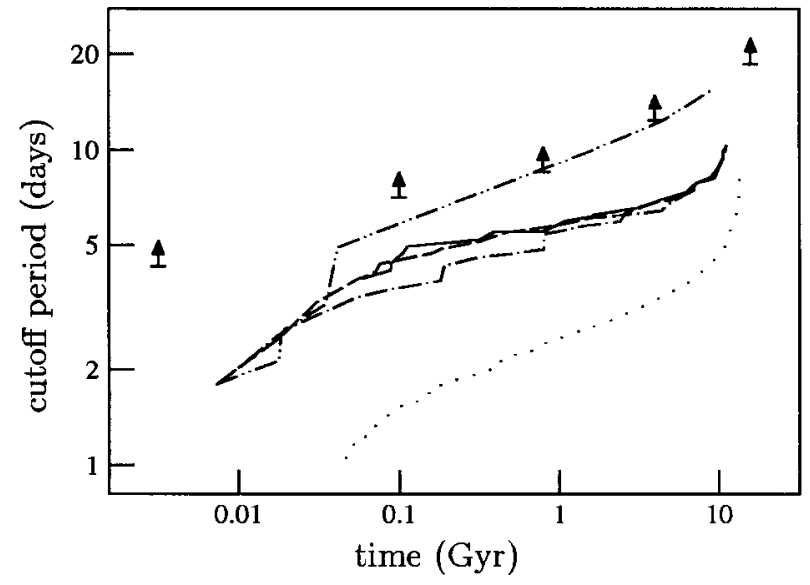

Fig. 10. Comparison of observed circularization cutoff periods (arrows) with theoretical calculations using Zahn's equilibrium tide theory (dotted curve), and with the dynamical tide theory including resonance locking for supersynchronous stars $\left(\Omega_{i}=\right.$ $2 \omega_{\text {per }}$, solid curve), quasi-synchronous stars $\left(\Omega_{i}=\omega_{\text {per }}\right.$, dashed curve), slowly rotating stars $\left(\Omega_{i}=\omega\right.$, dash-dot curve) and very slowly rotating stars $\left(P_{i}=100\right.$ days, dash-dot-dot curve). The dynamical tide yields closer agreement with the observations than the equilibrium tide.

theory of Zahn (1989), but disregarding possible pre-MS tidal evolution. All other curves correspond to calculations we performed for sets of stars with different initial rotation velocities and orbital periods and eccentricities between 0.1 and 0.8 on the ZAMS. The solid line gives the result for stars with initial fast rotation $\Omega_{i}=2 \omega_{\text {per }}$, the dashed line for stars with quasi-synchronous initial rotation $\left(\Omega_{i}=\omega_{\text {per }}\right)$, the dashed line with single dots for equal rotational and orbital periods $\left(\Omega_{i}=\omega\right)$, and the dashed line with double dots for slowly rotating stars with initially $P_{i}=100$ days. The circularization cutoff period is defined to be the largest orbital period for which a binary can become circularized down to $e \leq 0.01$. Pre-MS tidal evolution was not considered in the calculations, but the large discrepancy between the calculated and the observed cutoff periods near the onset of the MS in Fig. 10 indicates that for the closest binaries circularization does take place before the binary members settle on the ZAMS.

Depending on the initial rotation of the stars variations in the resulting orbital decay result. For the supersynchronous and quasi-synchronous stars (solid and dashed curves) similar cutoff periods are obtained, indicating that the stellar spin easily adjusts to the binary configuration, after which the tidal decay follows a typical evolution as described in Sect. 3.1.1. For very slowly rotating stars $q$ mode locking of harmonic $n=1$ as explained in Sect. 3.1.3 dominates the tidal evolution, preventing the star from spinning up towards (quasi-)synchronism, and resulting in a much larger cutoff period at a given age of the cluster (dash-dot-dot curve). For faster stellar rotation the relative forcing frequency of harmonic $n=1$ becomes higher retrograde, and for fast enough stellar rotation it becomes higher retrograde than the frequency of the fundamental $q$-mode ( $r$-mode). Identifying the frequency of the lowest radial order $q$-mode with the $r$-mode frequency $\bar{\sigma}_{q} \simeq-\frac{2 m}{l(l+1)} \Omega_{\mathrm{s}}$, with for the tidal $r$-modes $l=3$ (see Papaloizou \& Pringle 1978), we find for the critical stellar rotation $\sigma_{q}=\bar{\sigma}_{q}+m \Omega_{\mathrm{s}} \simeq\left(-\frac{1}{3}+2\right) \Omega_{\mathrm{s}}=\frac{5}{3} \Omega_{\mathrm{s}}=$ $n \omega \Rightarrow \Omega_{\mathrm{s}}=\frac{3 n}{5} \omega$. Locking of $n=1$ on $q$-modes can only occur if $\Omega_{\mathrm{s}} \lesssim \frac{3}{5} \omega$. Resonance locking on $q$-modes of harmonic $n=2$ can occur for stellar rotation speeds up to $\Omega_{\mathrm{s}} \lesssim \frac{6}{5} \omega$, and potentially such locking could result in enhanced decay of the eccentricity:

$\frac{\mathrm{d} e^{2}}{\mathrm{~d} t}=\frac{\omega \sqrt{1-e^{2}}}{E_{\text {orb }}}\left(n \sqrt{1-e^{2}}-m\right) \mathcal{T}_{n}$,

which for $n=m=2, e^{2}>0$ and negative $\mathcal{T}_{n}$ is negative. However, the calculations leading to the dash-dot curve in Fig. 10, for which the stellar rotation $\frac{3}{5} \omega<\Omega_{\mathrm{s}}=\omega<\frac{6}{5} \omega$ permits $q$-mode resonance locking for $n=2$, but not for $n=1$, show that this $n=2 q$-mode locking results in relatively slow orbital decay and therefore short cutoff periods. In systems with faster rotating stars $\left(\Omega_{\mathrm{s}}>\frac{6}{5} \omega\right)$ resonance locking of high frequency prograde modes against the forcing of many strong retrograde harmonics causes quicker orbital decay, while in systems with slower rotators $\left(\Omega_{\mathrm{s}}<\frac{3}{5} \omega\right)$ the efficient $n=1 q$-mode locking causes more rapid decay.

Regardless of stellar rotation, the dynamical tide with inclusion of the resonance locking mechanism predicts much stronger orbital decay than the equilibrium tide theory with viscous damping due to turbulent eddies. For the special case of very slowly rotating stars the calculations can even explain the observed cutoff periods for the stellar populations which have evolved well off the ZAMS. The case of binary stars which have virtually no rotation on the ZAMS may not be very common, however Bouvier et al. (1997) show that observations of G and K dwarf members of the Pleiades cluster do not indicate a relation between binarity and stellar rotation, and that the generally low rotation of single MS dwarfs in the Pleiades applies to binary members as well. These authors argue that accretion from a circumbinary disc during a large fraction of the pre-MS evolution may result in slowly rotating binary members. Projected rotation velocities as low as $v \sin i \approx 2 \mathrm{~km} \mathrm{~s}^{-1}$ are found, corresponding to a stellar rotation period $P_{\mathrm{s}} / \sin i \approx 25$ days.

Though we cannot exclude the possibility that some binary members of clusters do indeed contain very slowly rotating stars and therefore experience rapid orbital evolution due to $q$-mode locking, most of the binaries in clusters will probably not experience $q$-mode locking, and for these binaries there still seems to be a significant discrepancy between the observed and the theoretical cutoff periods. However, the observationally inferred cutoff periods do not come with a sound error estimate, and it is therefore not possible to evaluate the actual discrepancy.

Higher tidal decay rates could possibly be acquired by including Hansen coefficients of higher spherical degree $(l>2)$. Since all torques on the stars influence the rotation rates of the stars, all $l$-values are coupled in the 
balance of resonance locking. Inclusion of higher spherical degrees could thus result in additional resonance locking for $l>2$ harmonics, and in intensified locking of $l=2$ harmonics. In the same way, possible misalignment of the stellar rotation axes and the orbital angular momentum vector could result in more efficient resonance locking due to the action of the $l=2, m=1$ orbital harmonics which are of the leading order, and which in the case of misalignment do not vanish.

Other refinements which might lead to different tidal evolution rates include the effects of differential rotation, nonlinearity of the stellar response and resonant excitation of stellar modes with higher order latitudinal eigenvalues $\lambda_{n}$ (see SW02). Proper inclusion of these effects would however require substantial extra efforts to be made, the results of which can not easily be estimated beforehand.

\subsection{Planetary binaries}

Numerous planets outside our solar system with masses comparable to that of Jupiter and with orbital periods as short as 3 days have recently been discovered. Planet formation is not expected to be possible in such a tight orbit around a star, and while the inward migration of planets from the outer regions of a stellar system down to orbits with periods of a few days may be brought about by planet-disc or planet-planet interactions or by a second star passing by, once these tight orbits have been formed, tidal interaction with the central star may begin to play a role.

\subsubsection{Slow stellar rotation}

To determine how far the tidal influence of the central star reaches, we calculate orbital evolution of a planet with mass $M_{\mathrm{pl}}=\frac{1}{1000} M_{\odot}$ in a few weakly eccentric orbits $(e=0.1)$ around a slowly rotating $\left(P_{\mathrm{s}}=30\right.$ days $)$ solar mass star, with orbital periods of $P_{\text {orb }}=3,4,4.5,5$ and 6 days (see Figs. 11 and 12). It follows that the systems with initial orbital periods $P_{\text {orb }} \leq 4.5$ days can be circularized during the main sequence, while an orbit of $P_{\text {orb }}=5$ days goes through significant resonance locking without reaching circularization. The orbit with $P_{\text {orb }}=6$ days only experiences some tidal effects after stellar expansion near the end of the MS causes the relative separation at periastron $\left(D_{\text {per }} / R_{\mathrm{s}}=(1-e) a / R_{\mathrm{s}}\right.$, see panel $12 \mathrm{~d})$ to become less than 10 . For the system with $P_{\text {orb }}=6$ days, the stellar spin up due to tidal forcing is weaker than the stellar spin down due to the increasing stellar moment of inertia $I_{\mathrm{s}}$ as a result of nuclear evolution, therefore the star steadily spins down during the evolution (see panel 11c). Because the orbital and stellar moments of inertia are comparable, the frequency at which a neutral frequency shifting characteristic occurs $\left(\zeta_{\text {inn }}=0\right.$, dotted line labelled $\zeta_{0}$ in Fig. 12) is very low, $\sigma<2 \omega$. Because of this all the strong harmonics with $n \geq 2$ can cause locking while traversing a stellar resonance from the high frequency side to the low frequency side, contrary to what is usually seen in stellar binaries. And while in a stellar binary with a rapidly rotating star the retrograde forcing by the strong low frequency harmonics causes stellar spin down against which the higher harmonics may become resonance locked, in this planetary binary with a slowly rotating star the strongest harmonic $n=2$ is itself prograde, and the locking on this harmonic which is the main cause of the orbital decay is a result of the increasing eigenfrequencies of the stellar $g$-modes due to the restructuring of the star resulting from nuclear evolution. Note that the nature of a harmonic either being prone to cross or lock on to a resonance is sensitive to the mass of the planet; for higher mass planets the frequency for which $\zeta_{\text {inn }}=0$ goes up, possibly changing the nature of strong orbital harmonics from locking into non-locking.

\subsubsection{Fast stellar rotation}

If we want to consider planetary binaries with quickly rotating central stars, we must consider that the very small orbital moment of inertia, as compared to the case of a stellar binary, implies that the tidal spin down of the star may be much weaker than the spin down due to possible magnetic braking. We therefore show in Figs. 13 and 14 the evolution of a system with a planet in an orbit of $P_{\text {orb }}=5$ days and eccentricity $e=0.1$ around a star rotating at $15 \%$ of breakup ( $\simeq 16$ hours), for different values of the magnetic braking coefficient: $c_{\mathrm{mb}}=0,0.01,0.1$ and 1 . In the case without magnetic braking the stellar spin period is indeed hardly altered, while the cases with magnetic braking show much longer stellar spin periods.

In the calculation without magnetic braking the strong orbital harmonics remain highly retrograde throughout the orbital evolution, and numerous instances of resonance locking above the line $\zeta_{\text {inn }}=0$ (which jumps occasionally because of the different $n$-values of the harmonics that experience locking, see panel 14a) cause very significant expansion and decircularization of the orbit. In this case locking is driven by the increasingly negative eigenfrequencies of the modes due to nuclear evolution. Since both the direction of this frequency shift and the sign of the torque exerted by the modes changed with respect to the case of the slowly rotating star, resonance locking again occurs above the $\zeta_{0}$-line.

In the cases with increasingly stronger magnetic braking the retrograde $g$-modes are not strong enough to counter the downward shift of the eigenmodes imposed by the decelerating stellar spin, therefore these orbits initially do not change much while the star spins down. Eventually, however, the very strong $q$-modes are encountered, and these are strong enough to cause locking. For the case with strongest magnetic braking the initial spin down is very fast, and $q$-mode locking starts after only a very short while (panel $14 \mathrm{~d}$ ). This $q$-mode locking again causes very large orbital expansion and decircularization. For $c_{\mathrm{mb}}=0.01$ the locking only stars after $t=4 \mathrm{Gyr}$ 

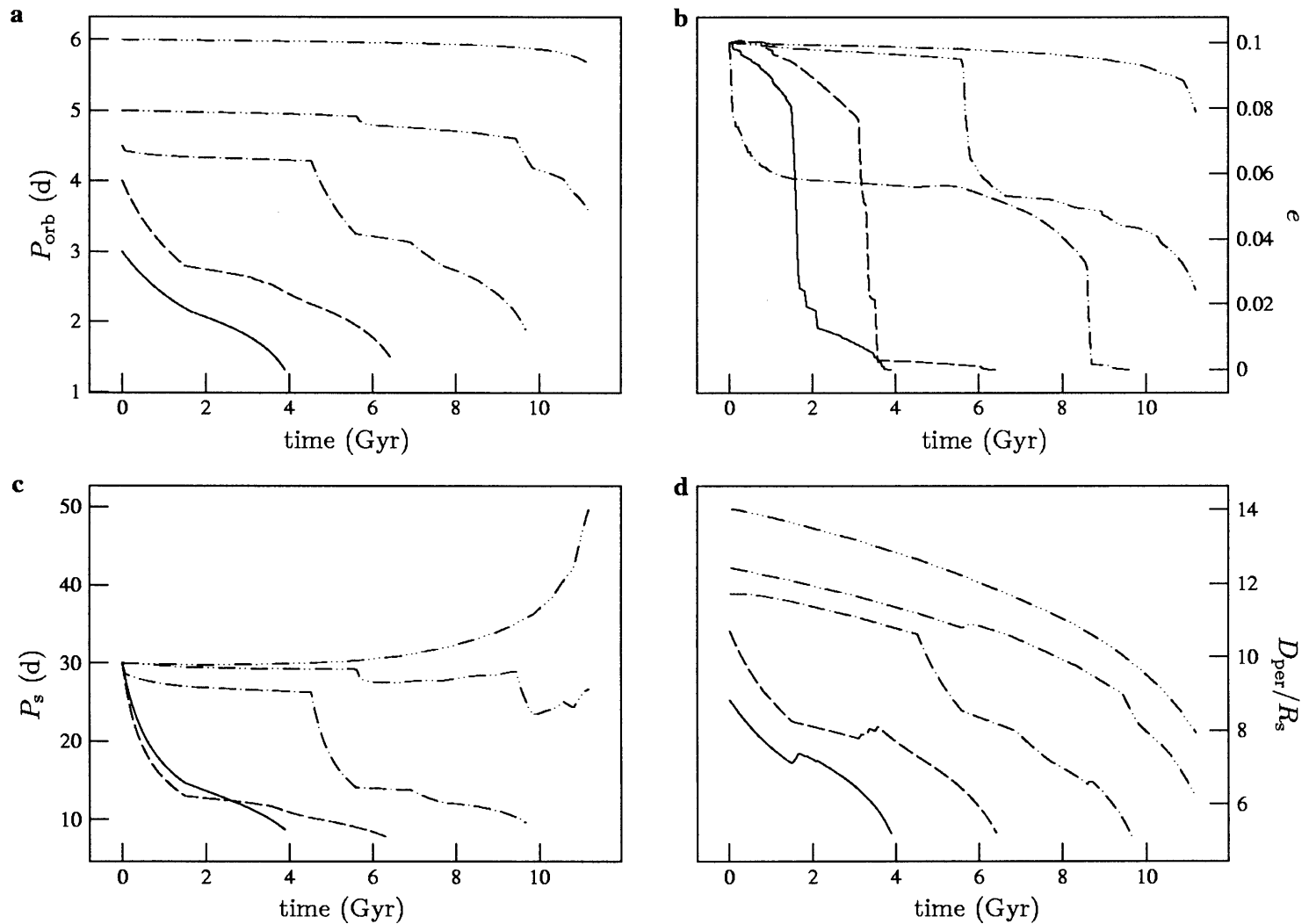

Fig. 11. Orbital evolution of a heavy planet around a slowly rotating solar type star. Tidal interaction works efficiently in orbits up to five days.
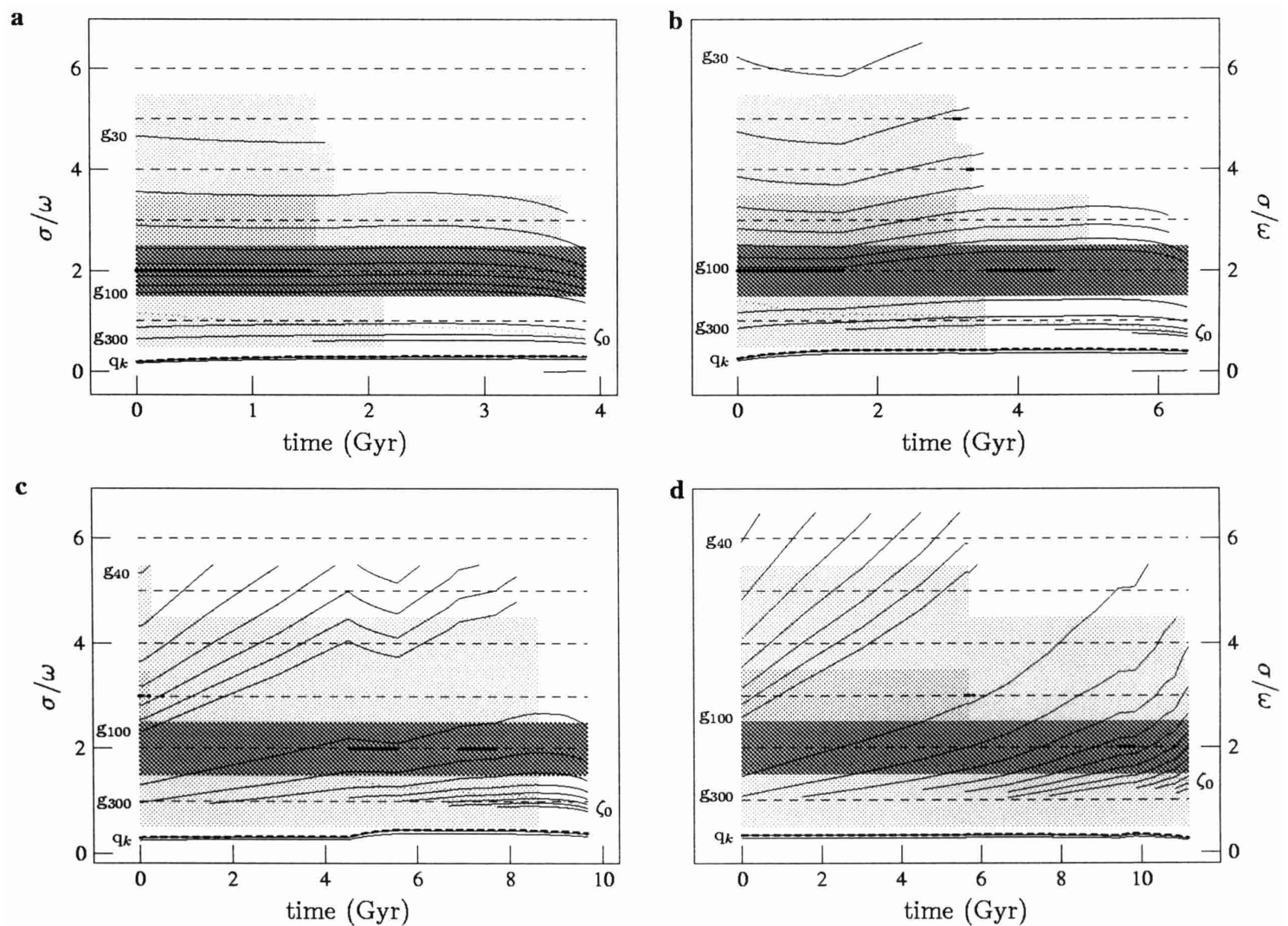

Fig. 12. Resonance locking for the systems in Fig. 11 with $P_{\text {orb }}=3,4,4.5$ and 5 days (panels a), b), c) and d), respectively). 

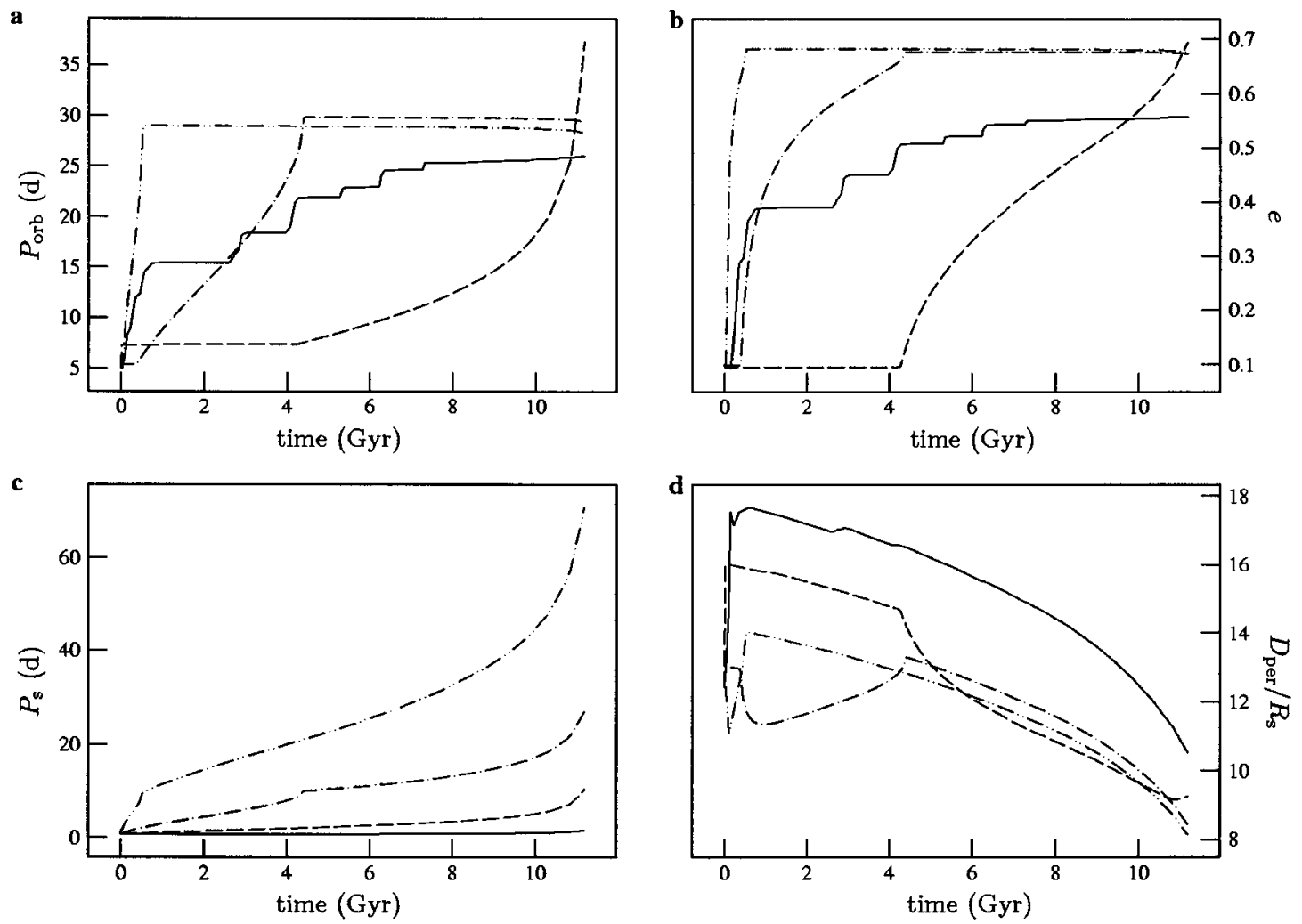

Fig. 13. Orbital evolution of a heavy planet around a quickly rotating solar type star with magnetic braking efficiency $c_{\mathrm{mb}}=0$ (solid line), $c_{\mathrm{mb}}=0.01$ (dashed line), $c_{\mathrm{mb}}=0.1$ (single dot) and $c_{\mathrm{mb}}=1$ (double dot). Retrograde resonance locking causes large expansion and decircularization of the orbits.
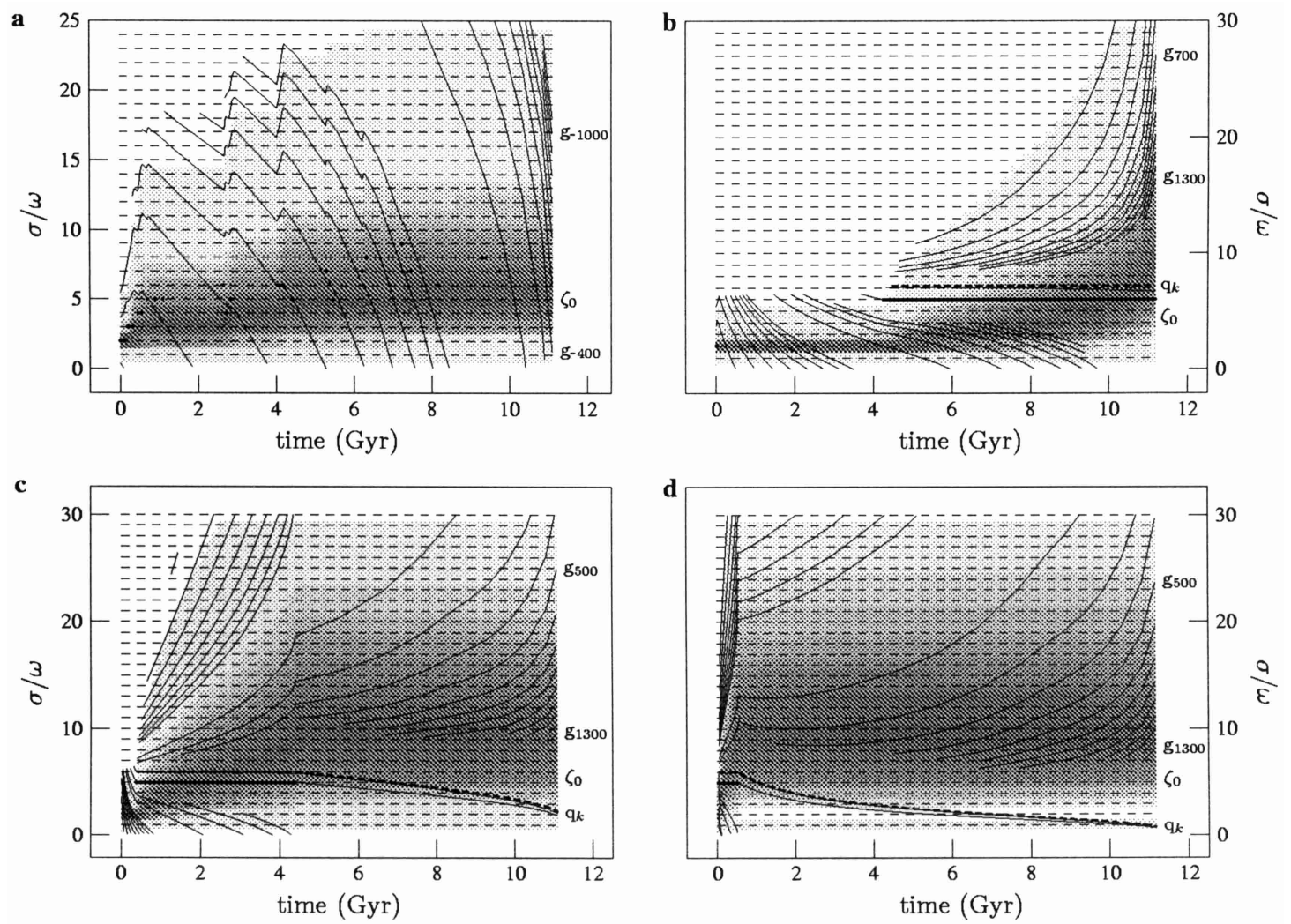

Fig. 14. Resonance locking for the planetary binaries with high stellar spin in Fig. 13 with the solid, dashed, single and double dot curves (panels a), $\left.\left.c_{\mathrm{mb}}=0 ; \mathbf{b}\right), c_{\mathrm{mb}}=0.01 ; \mathbf{c}\right), c_{\mathrm{mb}}=0.1$ and $\left.\mathbf{d}\right), c_{\mathrm{mb}}=1$, respectively). The large increase of the orbital eccentricity gives rise to a rapidly widening spectrum of orbital harmonics. 
and lasts for the remainder of the calculation (panel 14b), while for stronger braking the line $\zeta_{0}$ cuts through the locking harmonic $n=5$ after some time, disqualifying this harmonic for locking against modes shifting downwards in Fig. 14 (see panels c and d). Locking is then terminated, and the rapid orbital evolution halts.

In the end the different $q$-mode locked evolutionary paths result in similar eccentricities, while the retrograde $g$-mode locking produces a somewhat less eccentric orbit. The $g$-mode locking also results in the least wide orbit, while the weakest magnetic braking yields the largest orbital period.

The $q$-mode resonance locking in these planetary binaries is not terminated as a result of weakening of the exciting orbital harmonic or intrinsic weakening of the locked $q$-mode resonance, as might be the case in circularizing orbits or decelerating stars, but rather due to the changing character of the locked harmonic. At the onset of the locking the harmonic induces orbital and stellar spin changes which tend to keep the harmonic out of resonance, resulting in the locked situation. This tendency not to become resonant decreases and changes into the opposite, such that the locked harmonic induces orbital and stellar spin changes which drive itself closer to resonance. This result in a quick crossing of the resonance, terminating the resonance locking. Possible additional $q$-mode damping, leading to wider and lower $q$-mode torque peaks, is therefore not expected to change the orbital evolution.

\section{Conclusions}

We have calculated the tidal evolution of eccentric late type MS binary stars and of heavy planets which are in close eccentric orbit around a late type MS star. The tidal process which takes place in the solar type star is treated as a dynamical process, and close resonances between the forcing tidal harmonics and the eigenmodes of stellar $g$ and $q$-mode oscillation are taken into account. Due to the resonance locking mechanism, which causes extended intervals of close resonance, the dynamical tide is in solar type stars much more efficient than the equilibrium tide.

Cluster circularization cutoff periods up to 10 days near the end of the MS lifetime of the stellar binaries can be explained by the dynamical tide, while for the special case of very slow stellar rotation resonance locking on $q$ modes yields cutoff periods of up to 16 days. In this case of very slow stellar rotation close agreement with the observationally inferred relation between cutoff period and age of the stellar population (Mathieu et al. 1992) is acquired.

Weakly eccentric orbits of a Jupiter-like planet in orbit around a slowly rotating solar type star experience significant tidal decay due to resonance locking for orbital periods up to $\approx 5$ days. In case the star is quickly rotating, resonance locking causes strong orbital expansion and decircularization of an orbit with $P_{\text {orb }}=5$ days and $e=0.1$, resulting in orbital periods up to $P_{\text {orb }}=35$ days and eccentricities up to $e=0.7$, depending on the strength of magnetic braking in the star. The resonance locking, and therefore the orbital evolution, depends critically on the mass of the planet, because for higher planet masses the resonance locking characteristic of strong orbital harmonics swaps.

Acknowledgements. This work was sponsored by Spinoza grant 08-0 to E. P. J. van den Heuvel.

\section{References}

Bouvier, J., Rigaut, F., \& Nadeau, D. 1997, A\&A, 323, 139

Claret, A., \& Cunha, N. C. S. 1997, A\&A, 318, 187

Goodman, J., \& Dickson, E. S. 1998, ApJ, 507, 938

Goodman, J., \& Oh, S. P. 1997, ApJ, 486, 403

Mathieu, R. D., Duquennoy, A., Latham, D. W., et al. 1992, in Binaries as tracers of stellar formation, ed. A. Duquennoy, \& M. Mayor (Cambridge University Press), 278

Papaloizou, J., \& Pringle, J. E. 1978, MNRAS, 182, 423

Savonije, G. J., \& Papaloizou, J. C. B. 1983, MNRAS, 203, 581

Savonije, G. J., \& Papaloizou, J. C. B. 1997, MNRAS, 291, 633

Savonije, G. J., \& Witte, M. G. 2002, A\&A, 386, 211

Terquem, C., Papaloizou, J. C. B., Nelson, R. P., \& Lin, D. N. C. 1998, ApJ, 502, 788

Verbunt, F., \& Phinney, E. S. 1995, A\&A, 296, 709

Verbunt, F., \& Zwaan, C. 1981, A\&A, 100, L7

Witte, M. G., \& Savonije, G. J. 1999a, A\&A, 341, 842

Witte, M. G., \& Savonije, G. J. 1999b, A\&A, 350, 129

Witte, M. G., \& Savonije, G. J. 2001, A\&A, 366, 840

Zahn, J. P. 1977, A\&A, 57, 383

Zahn, J. P. 1989, A\&A, 220, 112

Zahn, J. P., \& Bouchet, L. 1989, A\&A, 223, 112 